\title{
Percent reduction of growth hormone levels correlates closely with percent resected tumor volume in acromegaly
}

\author{
Lucia Schwyzer, MD, Robert M. Starke, MD, John A. Jane Jr., MD, and Edward H. Oldfield, MD \\ Department of Neurological Surgery, University of Virginia Health System, Charlottesville, Virginia
}

OBJECT Correlation between tumor volume and hormone levels in individual patients would permit calculation of the fraction of tumor removed by surgery, by measuring postoperative hormone levels. The goals of this study were to examine the relationship between tumor volume, growth hormone (GH), and insulin-like growth factor-1 (IGF-1) levels, and to assess the correlation between percent tumor removal and the reduction in plasma GH and IGF-1 in patients with acromegaly.

METHODS The 3D region of interest-based volumetric method was used to measure tumor volume via MRI before and after surgery in 11 patients with $\mathrm{GH}$-secreting adenomas. The volume of residual tumor as a fraction of preoperative tumor volume was correlated with $\mathrm{GH}$ levels before and after surgery. Examination of this potential correlation required selection of patients with acromegaly who 1) had incomplete tumor removal, 2) had precise measurements of initial and residual tumor, and 3) were not on medical therapy.

RESULTS Densely granulated tumors produced more peripheral GH per mass of tumor than sparsely granulated tumors $(p=0.04)$. There was a correlation between GH and IGF-1 levels $(p=0.001)$. Although there was no close correlation between tumor size and peripheral GH levels, after normalizing each tumor to its own plasma GH level and tumor volume, a comparison of percent tumor resection with percent drop in plasma $\mathrm{GH}$ yielded a high correlation coefficient $(p=0.006)$.

CONCLUSIONS Densely granulated somatotropinomas produce more $\mathrm{GH}$ per mass of tumor than do sparsely granulated tumors. Each $\mathrm{GH}$-secreting tumor has its own intrinsic level of $\mathrm{GH}$ production per mass of tumor, which is homogeneous over the tumor mass, and which varies greatly between tumors. In most patients the fraction of a GH-secreting tumor removed by surgery can be accurately estimated by simply comparing plasma $\mathrm{GH}$ levels after surgery to those before surgery.

http://thejns.org/doi/abs/10.3171/2014.10.JNS14496

KEY WORDS acromegaly; growth hormone; tumor volume; pituitary surgery

$\mathrm{C}$ ERTAIN types of tumors, in the CNS and elsewhere, secrete substances that can be measured in the peripheral blood, and these biological markers routinely serve to screen, to diagnose, or to follow the course of the underlying disease. So far it has not been established if the fraction of tumor removal can be accurately determined by peripheral blood sampling. If there existed a close correlation between tumor mass and the serum level of a material secreted by the tumor, and if this substance could be easily and reliably measured in the peripheral blood, it would permit easy calculation of the remaining tumor mass after surgical removal or other ablative therapies.

Acromegaly is associated with considerable morbidity and mortality. First-line management is transsphenoidal tumor resection with the goal to normalize the growth hormone (GH) and insulin-like growth factor-1 (IGF-1) levels. ${ }^{8}$ However, since many of these tumors invade surrounding structures total resection cannot always be achieved. Hence, the hormone levels in these cases decrease after partial tumor removal, but do not become normal.

Although GH-secreting tumors produce levels of $\mathrm{GH}$ that can be easily measured in the peripheral blood, the correlation between tumor size and plasma GH levels varies widely from patient to patient. Furthermore, although there is a relationship between GH and IGF-1, it is nonlinear because of saturation of GH receptors when GH exceeds certain threshold levels, thresholds that also seem to vary widely from patient to patient. ${ }^{3}$

ABBREVIATIONS $\mathrm{GH}=$ growth hormone; IGF-1 = insulin-like growth factor-1.

SUBMITTED March 4, 2014. ACCEPTED October 9, 2014.

INCLUDE WHEN CITING Published online November 28, 2014; DOI: 10.3171/2014.10.JNS14496.

DISCLOSURE Dr. Schwyzer was supported by a postdoctoral fellowship grant from the Emil-Boral Foundation of Austria and Switzerland. 
To investigate this further, we examined various relationships between tumor size, plasma GH levels, and plasma IGF-1 before and after transsphenoidal surgery in patients with acromegaly caused by GH-secreting tumors.

We explored the possibility that if individual tumors have their own intrinsic level of GH production and if that level of GH production is homogeneous across the tumor, a comparison of GH levels before and after surgery would indicate the fraction of tumor that had been removed. Thus, a close correlation between tumor volume and hormone secretion in individual patients would permit calculation of the fraction of tumor removed by surgery, simply by measuring the postoperative $\mathrm{GH}$ levels.

\section{Methods}

We assessed the GH and IGF-1 levels before and after surgery in patients who were not on medical therapy and who had incomplete tumor removal, permitting the measurement of tumor mass before and after surgery. With approval from our local institutional review board, prospectively recorded medical records and imaging studies were systematically reviewed. Patients were excluded 1) if they had no visible residual tumor on postoperative MRI, because there could be no measurement of tumor size after surgery; and 2) if they were defined by the contemporary endocrine criteria as being in remission after surgery (IGF1 in the normal range for age and sex, glucose-suppressed plasma $\mathrm{GH}<1.0 \mathrm{ng} / \mathrm{ml}$ ), because the pulsatile nature of $\mathrm{GH}$ secretion after surgical remission makes random GH measurement unreliable. ${ }^{6}$ Patients underwent surgery between 2006 and 2009. The 3D region of interest-based volumetric method, in which the tumor margin is traced on each MRI slice and then the volume is calculated by using the area of each tumor on each slice and the slice thickness, was used to measure the tumor volume before and after surgery (NIH ImageJ) (http://rsbweb.nih.gov/ij/).

The values of GH and IGF-1 used were the last measurements before surgery (for GH 1-4 days before surgery, except in 1 patient it was 1 month before surgery; for IGF$1,1-3$ days before surgery, except in 2 patients it was 1 month before surgery); postoperative $\mathrm{GH}$ was obtained at 6-7:00 in the morning of the 1st day after surgery in all patients, except in 1 patient it was at 1 month, because no immediate postoperative $\mathrm{GH}$ was obtained. Because of its prolonged half-life of several weeks in the peripheral blood, postoperative IGF-1 was measured 2-3 months after surgery in all but 1 patient, in whom it was obtained 6 months after surgery. Tumor volumes were obtained on the last MRI before surgery (1 day -2 months before surgery) and on the first MRI obtained after surgery (2-3 months after surgery). Two patients did not have IGF-1 measurements while off medical therapy at 2-3 months after surgery. The GH and IGF-1 assays were as previously described. ${ }^{8}$

We examined correlations between tumor volume and plasma GH, tumor volume and IGF-1, tumor subtype (densely vs sparsely granulated GH-producing tumors) versus GH and IGF-1 levels in the peripheral blood, as well as the correlation between GH and IGF-1. The volume of residual tumor as a fraction of preoperative tumor volume was correlated with GH and IGF-1 levels before and after surgery, permitting normalization of hormone levels per mass of tumor for each patient.

\section{Statistical Analysis}

Comparison of tumor subtype with peripheral GH and IGF-1 levels was done with the Kruskal-Wallis test, and correlations of GH, IGF-1, tumor volume, and fraction of tumor removed versus fraction of change in GH and IGF-1 were analyzed with the Spearman rank correlation test $\left(\mathrm{r}_{\mathrm{s}}\right)$ using GraphPad Prism (GraphPad Software).

Measurement of the tumor volumes was done by L.S., who performed the measurements blinded to the GH or IGF-1 levels.

\section{Results}

There were 11 patients who met the criteria for study among 96 patients who had surgery for acromegaly between 2006 and 2009.

Although there was a trend toward correlation between tumor size and $\mathrm{GH}$, it was not significant $(\mathrm{p}=0.07)$; there was a correlation between tumor volume and plasma IGF$1\left(r_{s}=0.64, p=0.002\right.$; Fig. 1). There was also a correlation when comparing plasma GH versus plasma IGF-1 $\left(\mathrm{r}_{\mathrm{s}}\right.$ $=0.66, \mathrm{p}=0.001$; Fig. 2). Comparison of percent tumor resection and percent drop in IGF-1 did not demonstrate a correlation ( $p=0.42$; Fig. 3$)$. Despite that there was no significant correlation between tumor size and GH levels, comparison of percent tumor resection with percent drop in plasma $\mathrm{GH}$, thus normalizing each tumor to its own initial volume and plasma GH level, yielded a high correlation $\left(r_{s}=0.78, p=0.006\right.$; Fig. 3$)$.

Densely granulated tumors were associated with greater values for peripheral GH levels in comparison with sparsely granulated tumors (Kruskal-Wallis, $p=0.04$ ); there was a trend for the same with IGF-1 levels, but it did not reach statistical significance ( $\mathrm{p}=0.3$; Fig. 4).

\section{Discussion}

Because plasma IGF-1 has a long half-life it has a relatively steady level in normal subjects and in patients with acromegaly. Thus, for diagnosing acromegaly, measurement of IGF-1 is more reliable than measurement of GH because of the random pulsatile nature of GH secretion. In acromegaly the pituitary tumor produces $\mathrm{GH}$, whereas IGF-1 is produced in the liver in response to plasma GH; IGF-1 has a sigmoid-shaped relationship with GH as a result of the hormone-receptor interactions between $\mathrm{GH}$ and IGF-1, with IGF-1 reaching a plateau after GH levels have reached levels sufficient to saturate GH receptors. Studies in which frequent sampling of GH is performed indicate that although GH is secreted in pulses in patients with acromegaly, as it is in normal subjects, the secretory bursts in acromegaly are much less prominent in comparison with the elevated basal GH concentration. ${ }^{6,7,10}$ Thus, most random measurements of $\mathrm{GH}$ in patients with acromegaly reflect the relatively steady basal GH level, rather than a level associated with a secretory pulse. Since GH is produced by the tumor it seems logical that the plasma GH 
GH vs. Tumor volume

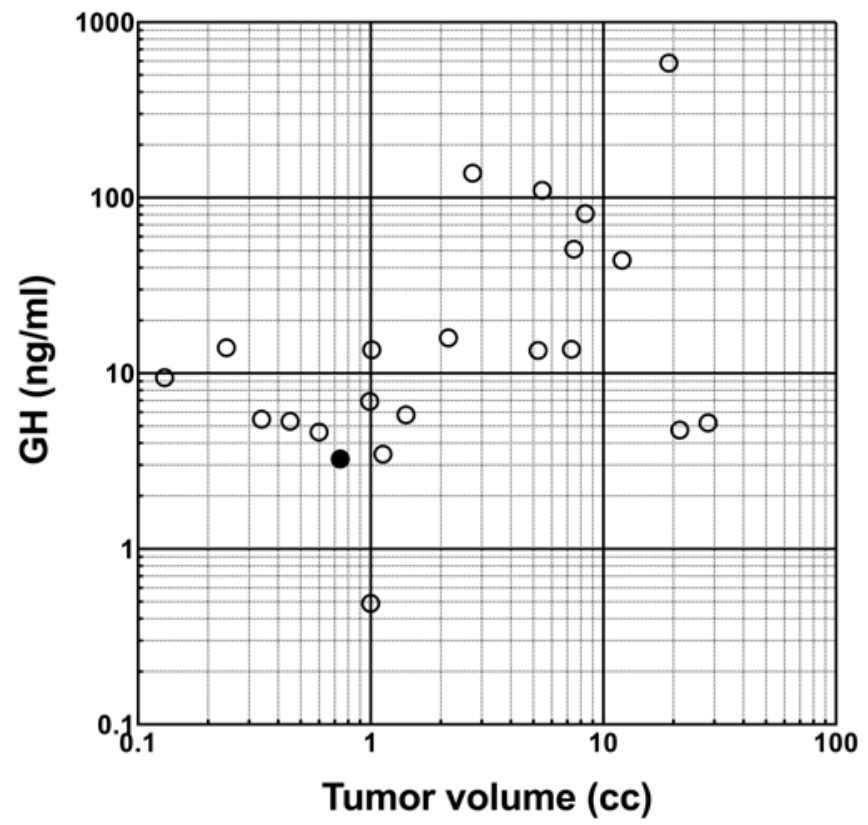

IGF-1 vs. Tumor volume

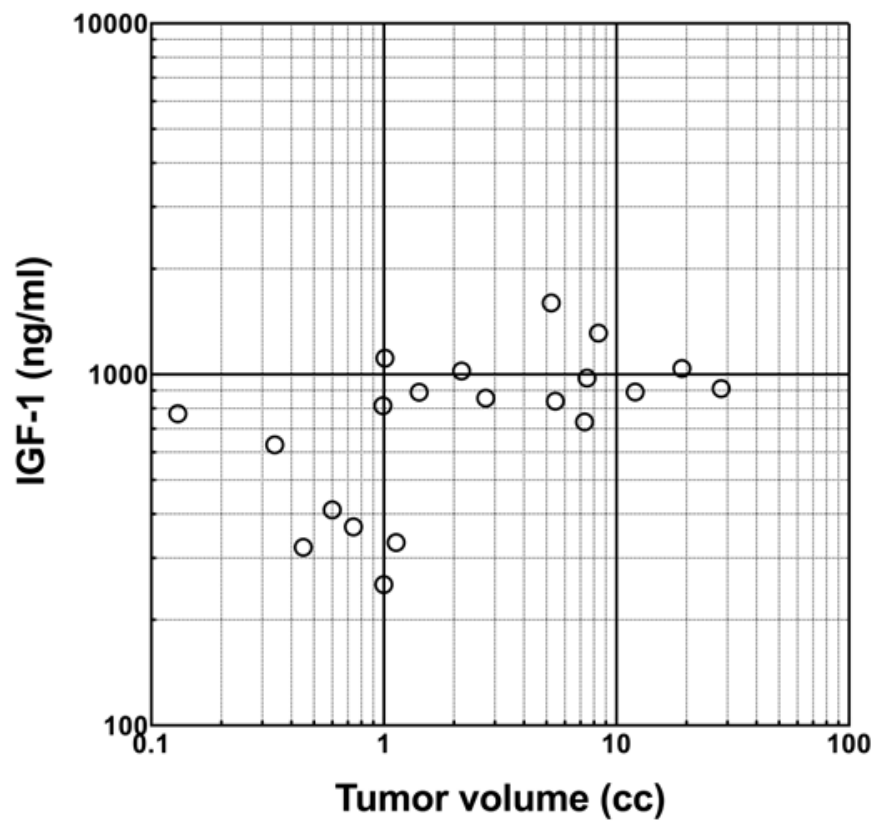

FIG. 1. Graphs showing a trend toward a correlation when comparing tumor volume to plasma GH (left; $p=0.07$ ). The levels of plasma IGF-1 correlated with tumor volume (right; $r_{s}=0.64, p=0.002$ ). Note that the measurements include the volumes measured before and after surgery. The black closed circle in Figs. 1-3 represents the $\mathrm{GH}$ level of a patient whose $\mathrm{GH}$ was measured 1 month after surgery. All other postoperative $\mathrm{GH}$ measurements were made in the morning of the 1st day after surgery.

levels would correlate with the tumor size in individual patients, although if different tumors have different potency of GH production the slope of that correlation would differ from patient to patient.

Based on the cellular distribution of GH staining with immunohistochemistry and their appearance with electron microscopy, GH-producing tumors are classified as densely granulated, sparsely granulated, or as an intermediate, mixed group. Assessment of our small set of tumors indicates that the densely granulated tumors produce higher levels of plasma GH per unit of tissue volume than do sparsely granulated tumors. A similar trend occurred



FIG. 2. Graph showing a correlation between plasma $\mathrm{GH}$ versus plasma IGF-1 $\left(r_{s}=0.66, p=0.001\right)$. The values plotted include the measurements before and after surgery. One GH value of $568 \mathrm{ng} / \mathrm{ml}$ is not included in the graph. with plasma IGF-1 levels per unit of tumor volume. This is in agreement with the recent findings of Fougner et al., who in their recent analysis of 78 tumors concluded that densely granulated tumors produce more IGF-1 per unit of tumor volume than do sparsely granulated tumors. ${ }^{5}$ They noted a similar trend for $\mathrm{GH}$, although it did not reach significance. Obari et al. had previously noted higher mean plasma GH levels in patients with densely granulated and mixed tumors compared with patients with sparsely granulated tumors, and Bakhtiar et al. had previously found that the GH-producing index (peripheral GH levels per cubic centimeter of tumor) was lower in sparsely granulated compared with densely granulated tumors.,12

That the percent reduction in GH levels after surgery is tightly linked to the percent of tumor that was resected has potential practical relevance. For instance, the success of radiation therapy and medical therapy for GH-producing tumors is related to the degree of pretreatment elevation of GH. ${ }^{2,4,9,11,13}$ Occasionally, preoperative imaging suggests that a tumor cannot be totally removed with surgery, but predicts that the fraction of tumor that can probably be safely removed would produce GH levels that would yield higher chances of achieving a normal GH and IGF-1 level with subsequent medical or irradiation therapy, and would permit earlier return to normal levels of GH and IGF-1 after adjuvant therapy. Knowing that the percent reduction in $\mathrm{GH}$ can be reasonably predicted by the fraction of tumor that can probably be removed surgically might provide information on whether to suggest additional surgery, or not.

The limitation of our study is the relatively small number of patients. However, to address the issue that we wished to address required that patient selection include 

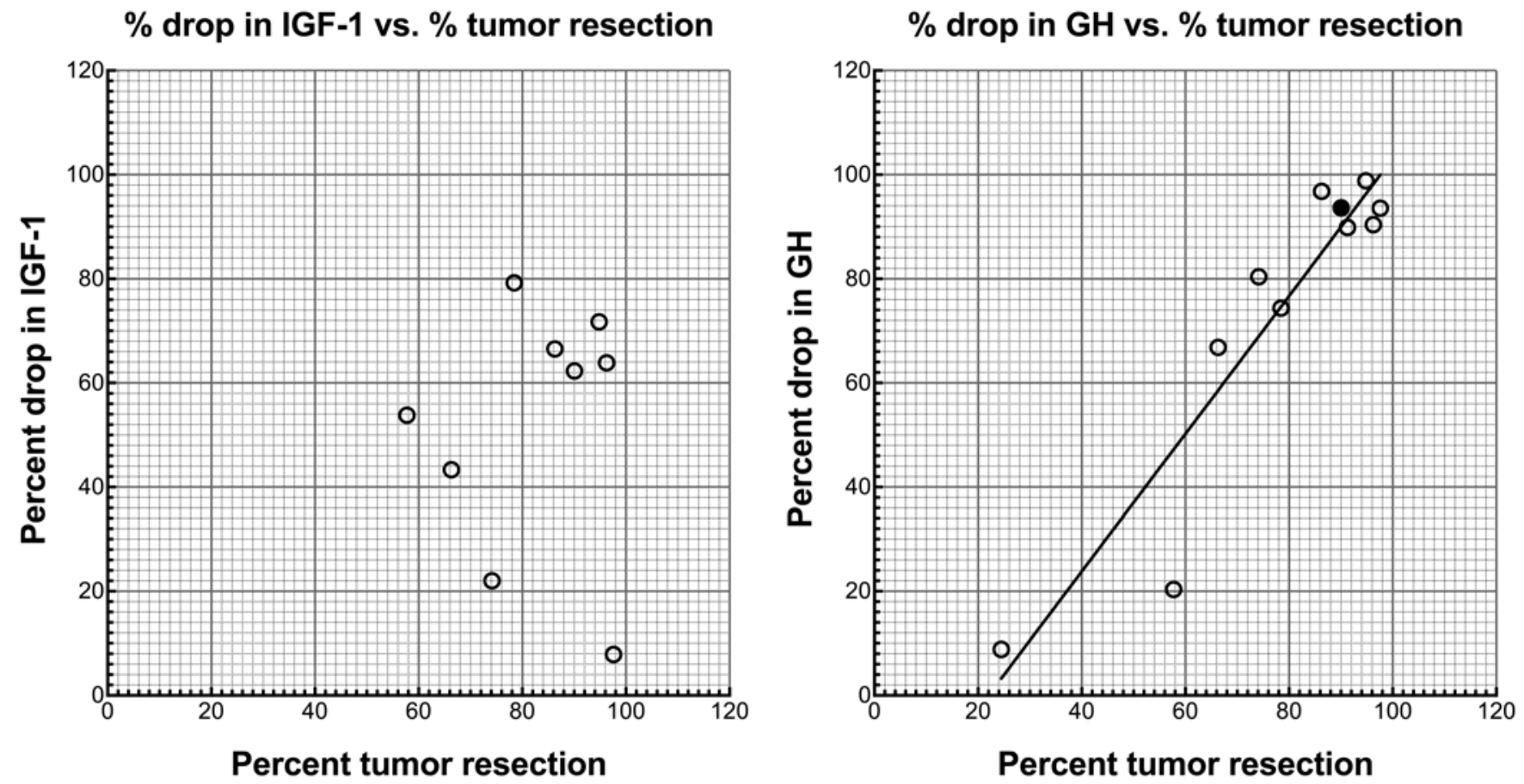

FIG. 3. Graphs showing that there was no correlation between the percent drop in plasma IGF-1 and the percent tumor removal (left; $p=0.42$ ). However, a comparison of percent tumor resection with percent drop in plasma $\mathrm{GH}$, permitting normalization of each tumor to its own plasma GH level, yielded a high correlation coefficient (right; $r_{s}=0.78, p=0.006$ ).

\section{GH Levels vs. Tumor type}



IGF-1 Levels vs. Tumor type

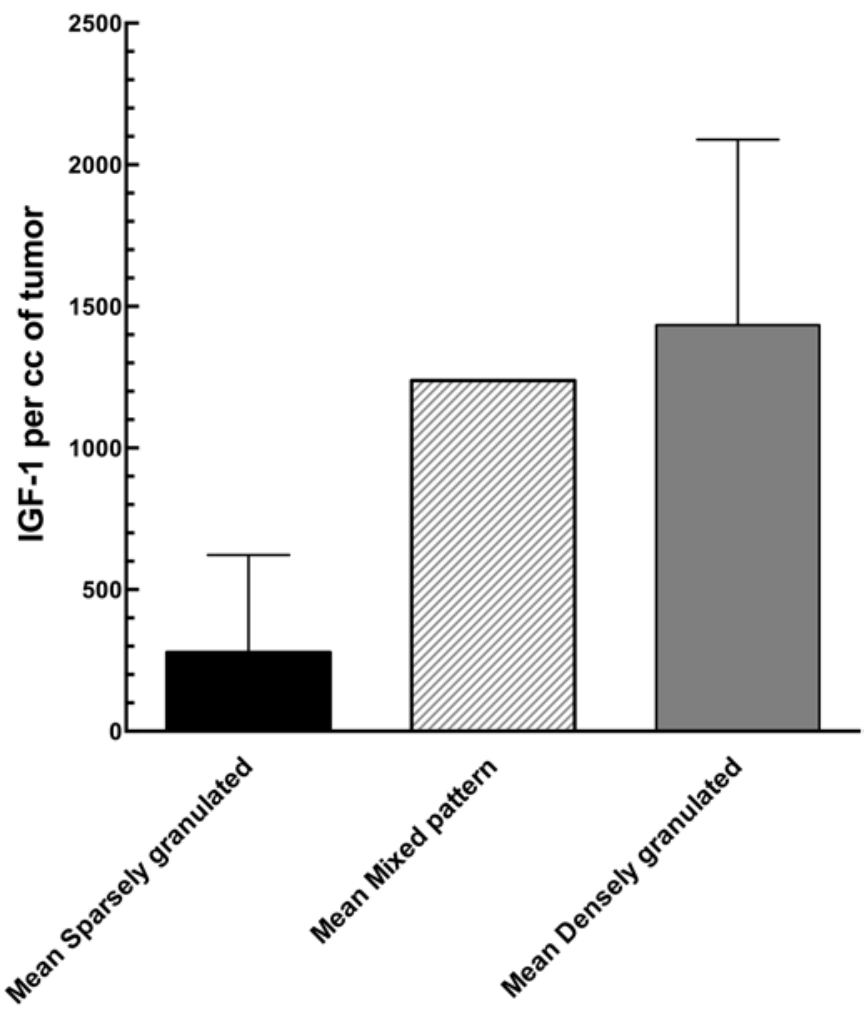

FIG. 4. Bar graphs showing that densely granulated tumors $(n=4)$ produced higher levels of plasma GH (left; Kruskal-Wallis, $p$ $=0.04$ ) and IGF-1 than sparsely granulated tumors $(n=6)$, although the difference did not reach statistical significance for IGF-1 (right; $p=0.3$ ). The values shown represent the means of the average GH and IGF-1 levels per cc of tumor as measured before and after surgery. There was 1 mixed tumor. The error bars represent SEMs. 
only those with partial tumor removal that could be imaged using MRI, in patients who were not receiving medical therapy during imaging performed before and after surgery.

\section{Conclusions}

These results indicate that each $\mathrm{GH}$-secreting tumor has its own intrinsic level of GH production per mass of tumor, that that level is homogeneous over the tumor mass, and that this varies greatly between tumors. Our study further indicates that the fraction of a GH-secreting tumor incompletely removed by surgery can be accurately estimated simply by comparing plasma GH levels after surgery to those before surgery. The same principles will likely apply to other types of tumors that produce hormones or other products that can be measured in the peripheral blood, although we have been unable to find a similar analysis in any other tumor type.

\section{References}

1. Bakhtiar Y, Hirano H, Arita K, Yunoue S, Fujio S, Tominaga A, et al: Relationship between cytokeratin staining patterns and clinico-pathological features in somatotropinomae. Eur J Endocrinol 163:531-539, 2010

2. Bevan JS, Atkin SL, Atkinson AB, Bouloux PM, Hanna F, Harris PE, et al: Primary medical therapy for acromegaly: an open, prospective, multicenter study of the effects of subcutaneous and intramuscular slow-release octreotide on growth hormone, insulin-like growth factor-I, and tumor size. J Clin Endocrinol Metab 87:4554-4563, 2002

3. Clemmons DR, Van Wyk JJ, Ridgway EC, Kliman B, Kjellberg RN, Underwood LE: Evaluation of acromegaly by radioimmunoassay of somatomedin-C. N Engl J Med 301:1138-1142, 1979

4. Colao A, Attanasio R, Pivonello R, Cappabianca P, Cavallo LM, Lasio G, et al: Partial surgical removal of growth hormone-secreting pituitary tumors enhances the response to somatostatin analogs in acromegaly. J Clin Endocrinol Metab 91:85-92, 2006

5. Fougner SL, Casar-Borota O, Heck A, Berg JP, Bollerslev J: Adenoma granulation pattern correlates with clinical variables and effect of somatostatin analogue treatment in a large series of patients with acromegaly. Clin Endocrinol (Oxf) 76:96-102, 2012

6. Hartman ML, Pincus SM, Johnson ML, Matthews DH, Faunt LM, Vance ML, et al: Enhanced basal and disorderly growth hormone secretion distinguish acromegalic from normal pulsatile growth hormone release. J Clin Invest 94:1277-1288, 1994
7. Hartman ML, Veldhuis JD, Vance ML, Faria AC, Furlanetto RW, Thorner MO: Somatotropin pulse frequency and basal concentrations are increased in acromegaly and are reduced by successful therapy. J Clin Endocrinol Metab 70:13751384,1990

8. Jane JA Jr, Starke RM, Elzoghby MA, Reames DL, Payne $\mathrm{SC}$, Thorner MO, et al: Endoscopic transsphenoidal surgery for acromegaly: remission using modern criteria, complications, and predictors of outcome. J Clin Endocrinol Metab 96:2732-2740, 2011

9. Jenkins PJ, Bates P, Carson MN, Stewart PM, Wass JAH: Conventional pituitary irradiation is effective in lowering serum growth hormone and insulin-like growth factor-I in patients with acromegaly. J Clin Endocrinol Metab 91:1239-1245, 2006

10. Kaltsas GA, Isidori AM, Florakis D, Trainer PJ, CamachoHubner C, Afshar F, et al: Predictors of the outcome of surgical treatment in acromegaly and the value of the mean growth hormone day curve in assessing postoperative disease activity. J Clin Endocrinol Metab 86:1645-1652, 2001

11. Karavitaki N, Turner HE, Adams CB, Cudlip S, Byrne JV, Fazal-Sanderson V, et al: Surgical debulking of pituitary macroadenomas causing acromegaly improves control by lanreotide. Clin Endocrinol (Oxf) 68:970-975, 2008

12. Obari A, Sano T, Ohyama K, Kudo E, Qian ZR, Yoneda A, et al: Clinicopathological features of growth hormoneproducing pituitary adenomas: difference among various types defined by cytokeratin distribution pattern including a transitional form. Endocr Pathol 19:82-91, 2008

13. Roth J, Gorden P, Brace K: Efficacy of conventional pituitary irradiation in acromegaly. N Engl J Med 282:1385-1391, 1970

\section{Author Contributions}

Conception and design: Oldfield, Schwyzer. Acquisition of data: Oldfield, Schwyzer, Jane. Analysis and interpretation of data: Oldfield, Schwyzer, Starke. Drafting the article: Oldfield, Schwyzer, Jane. Critically revising the article: Oldfield, Starke. Reviewed submitted version of manuscript: all authors. Approved the final version of the manuscript on behalf of all authors: Oldfield. Statistical analysis: Oldfield, Starke. Administrative/ technical/material support: Oldfield, Jane. Study supervision: Oldfield.

\section{Correspondence}

Edward H. Oldfield, Department of Neurological Surgery, University of Virginia Health System, Box 800212, Charlottesville, VA 22908-0212. email: eho4u@virginia.edu. 